\title{
Giardia lamblia infection increases risk of chronic gastrointestinal disorders
}

\author{
Megan Dormond ${ }^{1,2}$, Ramiro L. Gutierrez ${ }^{1}$ and Chad K. Porter ${ }^{1 *}$ (D)
}

\begin{abstract}
Background: Giardia lamblia is a common parasitic cause of infectious gastroenteritis in the United States and the world and may be linked to an increased risk of chronic gastrointestinal (Gl) disorders. We sought to assess the risk of several chronic GI disorders following Giardia infection among active duty US military personnel.

Methods: This study was designed as a retrospective cohort study in which active duty military personnel with documented G. lamblia infection were assessed for the subsequent risk of developing a chronic Gl disorder including irritable bowel syndrome (IBS), dyspepsia and gastroesophageal reflux disease (GERD). Post-giardia chronic Gl disorder risk was compared to risk in uninfected personnel matched on several demographic characteristics and medical encounter information. Data were obtained from the Defense Medical Surveillance System and exposures (1998-2009) with outcomes identified based on documented medical encounters with specific medical billing codes. Modified Poisson regression was used to evaluate the relationship between G. lamblia infection and chronic Gl disorders.
\end{abstract}

Results: A total of 80 Giardia cases were identified for an estimated incidence of 0.55 cases per 100,000 person-years. Cases were matched to 294 unexposed subjects. After adjusting for important covariates, there was an increased risk of IBS (relative risk: 2.1, $p=0.03$ ) associated with antecedent Giardia infection.

Conclusion: These data add to a growing body of literature and demonstrate an increased risk of IBS after infection with G. lamblia.

Keywords: Functional gastrointestinal disorders, Giardia lamblia, Post infectious, Infectious gastroenteritis

Abbreviations: FGD, Functional gastrointestinal disorder; GERD, Gastroesophageal reflux disease; Gl, Gastrointestinal; IBS, Irritable bowl syndrome

\section{Background}

Infectious gastroenteritis (IGE) is caused by numerous bacterial, viral, and parasitic pathogens. Among parasites, Giardia lamblia, a unicellular eukaryotic microorganism that causes symptoms of diarrhea, abdominal cramps, and occasionally nausea and vomiting, is a predominate pathogen. Symptoms of giardiasis usually occur within 1-2 weeks following exposure; however, they can persist for weeks or even months without proper treatment [1]. Giardia accounts for approximately 77,000 cases of parasitic foodborne illness annually in the US [2] and is a known cause of travelers' diarrhea among civilian and military

\footnotetext{
* Correspondence: chad.k.porter2.mil@mail.mil

${ }^{1}$ Enteric Disease Department, Naval Medical Research Center, 503 Robert Grant Avenue, Silver Spring, MD 20910, USA

Full list of author information is available at the end of the article
}

travelers $[3,4]$. Globally, the burden is even greater with recent literature from the Foodborne Disease Burden Epidemiology Reference Group (FERG) estimating 183 million cases and 171,000 disability adjusted life years (DALYs) attributed to G. lamblia in 2010 [5].

In addition to the acute illness, giardiasis has been associated with an increased risk of several secondary or chronic health conditions and is known to cause small intestinal malabsorption [1]. One such group of sequelae that has been increasingly associated with other enteric pathogens are functional gastrointestinal disorders (FGD). The pathophysiology of FGDs is likely varied and has yet to be clearly characterized but is typified by the onset of chronic or recurrent GI symptoms [6]. The FGDs have been categorized into 24 separate diagnoses based on the "Rome Diagnostic Criteria" [7, 8]. As a 
group, these disorders are estimated to account for more than 11 million ambulatory care visits, equating to roughly four visits for every 100 persons in the United States [8]. The pathoetiology of these disorders is poorly understood; however, several mechanisms have been implicated in producing symptoms including gut brain axis dysfunction, mucosal barrier disruption, gastrointestinal dysmotility, microbiota disturbances, inflammation, visceral hypersensitivity, diet, and genetic predisposition [8].

We sought to assess the association between G. lamblia infection and several FGD in active duty US military personnel, a healthy subset of the general travel population. The outcomes studied have been previously shown to be associated with infectious gastroenteritis [8-12]; however, data linking antecedent Giardia infections are lacking.

\section{Methods}

This retrospective cohort study evaluated the risk of PI sequelae following a documented parasitic IGE episode compared to the risk of those same outcomes in a cohort of unexposed subjects. Exposed subjects were identified by the International Classification of Diseases, volume 9 - Clinical Modification (ICD9-CM) codes using the Defense Medical Surveillance System (DMSS). Giardia exposure was identified based on a documented medical encounter with 007.1 visit code. Up to four unexposed subjects were matched to each Giardia case based on an unrelated medical encounter within 1 year of the Giardia exposure exposed. Unrelated medical encounters codes included the following: acute respiratory infections (ICD9-CM: 460-466), pneumonia and influenza (ICD9-CM: 480-488), infectious of the skin and subcutaneous tissue (ICD9-CM: 680-686), dislocations (ICD9-CM: 830-839), sprains and strains for joints and adjacent muscles (ICD9-CM: 840-848), burns (ICD9CM: 910-949), fracture of upper or lower limb (ICD9CM: 810-829). Additional matching criteria included age ( \pm 1 year), gender, number of prior deployments, medical treatment facility and type of medical encounter (inpatient or outpatient). All subjects had a minimum of 1 year of documented follow-up within the DMSS and were selected from the total active duty US military population from 1998 to 2009. The outcomes of interest were irritable bowel syndrome (IBS) (ICD9-CM: 564.1, 306.4), constipation (ICD9-CM: 564.0), dyspepsia (ICD9-CM: 536.8) and gastroesophageal reflux disease (GERD) (ICD9-CM: 530.81).

Additional covariates were included to determine their effect on the risk of PI sequelae. ICD9-CM codes for Axis I psychological disorders of interest included neurotic disorders (300), personality disorders (301), sexual disorders (302), acute reaction to stress (308), adjustment reaction
(309), non-psychotic brain syndrome (310), depressive disorders not elsewhere classified (311), conduct disturbances not elsewhere classified (312), emotional disturbance (313), and hyperkinetic syndrome (314).

Incidence of Giardia was estimated based on the number of cases identified and the total active duty military population data obtained from the Defense Manpower Data Center. Descriptive characteristics were calculated and stratified by exposure. Associations between the exposure and outcomes were initially explored using appropriate univariate methods. Modified Poisson regression models using a robust sandwich estimator for variance were used to investigate the relationship between giardiasis and each of the outcomes independently [13]. Multivariate models were built for each outcome using backwards elimination and an alpha $=0.15$ to retain variables. All statistical analyses were conducted using SAS v. 9.3 for Windows (SAS Institute, Cary, North Carolina).

\section{Results}

A total of 80 giardiasis cases were identified for an estimated incidence of 0.55 per 100,000 person-years in males (no female cases were identified). Giardiasis cases were matched to 294 unexposed subjects for a total sample size of 374 subjects. All of subjects were male with a median age of 35 years (interquartile range: 32, 46) at the time of study completion (Table 1). Over two-thirds $(n=272)$ of the study population had at least a high school education while the remaining $(n=65)$ had either bachelor's $(n=31,8.2 \%)$ or master's degrees $(n=34$, $11.6 \%$ ) (Table 1). Occupation was fairly evenly distributed among all the possible categories. In terms of branch of service, many of the study subjects were in the Navy (31.1 \%) followed closely by the Army (29.4\%) and finally the Marines (22.2 \%) (Table 1).

In univariate analyses (Table 2), G. lamblia infection was associated with an increased risk of IBS (relative risk $\{R R\}$ [10]: 2.1; $95 \%$ confidence interval $\{C I\}: 1.1,3.7)$ and dyspepsia (RR: 3.2; 95 \% CI: 1.2, 8.9) compared to unexposed subjects (Table 2). Those individuals that were diagnosed with an Axis I disorder were also at a 6.7 times (95 \% CI: 3.7, 12.2) increased risk for IBS. GERD (4.0, 95 \% CI: 2.9, 5.6), and dyspepsia (RR: 5.4, 95 \% CI: 1.7, 16.5) were also significantly associated with Axis I disorders. Other bacterial and viral IGE episodes were associated with the development of IBS (RR: 5.9, $95 \%$ CI: 3.2, 10.8), GERD (RR: $2.1,95 \%$ CI: 1.3, 3.5) and functional constipation (RR: 9.0, $95 \%$ CI: 2.7, 29.8).

In multivariate models (Table 3 ), the risk of all outcomes remained elevated in those with Axis I disorders with adjusted $R R\{a R R\}$ ranging from 3.4 to 12.5 . Individual disorders were not investigated independently due to low numbers of each diagnosis. In contrast, military rank and race appeared to have differential effects across 
Table 1 Demographic characteristics of selected US military members between 1998-2009

\begin{tabular}{|c|c|c|c|}
\hline & G. lamblia & Unexposed & Total \\
\hline $\bar{N}$ & 80 & 294 & 374 \\
\hline Age [Median (IQR)] & $37.35(34,45)$ & $35(30,46)$ & $35(32,46)$ \\
\hline \multicolumn{4}{|l|}{$\operatorname{Sex}[n(\%)]$} \\
\hline Male & $80(100)$ & $294(100)$ & $374(100)$ \\
\hline \multicolumn{4}{|l|}{ Race/Ethnicity [n (\%)] } \\
\hline Hispanic & $13(16.3)$ & $69(23.5)$ & $82(23.7)$ \\
\hline White & 67 (83.8) & $215(73.1)$ & $282(75.4)$ \\
\hline Other/Unknown & $0(0)$ & $10(3.4)$ & $10(2.6)$ \\
\hline \multicolumn{4}{|l|}{ Education [n (\%)] } \\
\hline High School & $55(68.8)$ & $217(73.8)$ & $272(72.7)$ \\
\hline Some College & $10(12.5)$ & $25(8.5)$ & $35(9.4)$ \\
\hline College & $5(6.3)$ & $26(8.8)$ & $31(8.2)$ \\
\hline Graduate School & $10(12.5)$ & $24(8.2)$ & $34(11.6)$ \\
\hline Unknown & $0(0)$ & $1(0.3)$ & $1(0.3)$ \\
\hline \multicolumn{4}{|l|}{ Rank [n (\%)] } \\
\hline Junior enlisted & $23(28.8)$ & $116(39.5)$ & $139(50.7)$ \\
\hline Senior enlisted & $44(55.0)$ & $130(44.2)$ & $174(46.5)$ \\
\hline Junior officer & $0(0)$ & $2(0.7)$ & $5(0.8)$ \\
\hline Senior officer & $13(16.3)$ & $46(15.6)$ & $59(15.7)$ \\
\hline \multicolumn{4}{|l|}{ Service $[\mathrm{n}(\%)]$} \\
\hline Army & $29(36.3)$ & $81(27.5)$ & $110(29.4)$ \\
\hline Coast Guard & $0(0)$ & $12(4.1)$ & $12(3.2)$ \\
\hline Air force & $12(15)$ & $41(13.9)$ & $53(14.2)$ \\
\hline Marines & $13(16.3)$ & $70(23.8)$ & $83(22.2)$ \\
\hline Navy & $26(32.5)$ & $90(30.6)$ & $116(31.1)$ \\
\hline \multicolumn{4}{|l|}{ Military Operation [n (\%)] } \\
\hline Iraqi Freedom (Iraq) & $17(21.24)$ & $85(28.9)$ & $102(76.7)$ \\
\hline $\begin{array}{l}\text { Enduring Freedom } \\
\text { (Afghanistan) }\end{array}$ & $8(10.0)$ & 49 (16.6) & $57(65.4)$ \\
\hline \multicolumn{4}{|l|}{ Primary Outcomes } \\
\hline Dyspepsia & $8(10.0)$ & $5(17)$ & $13(3.5)$ \\
\hline Constipation & $4(5.0)$ & $6(20.4)$ & $10(2.6)$ \\
\hline IBS & $16(20.0)$ & $16(5.4)$ & $32(8.5)$ \\
\hline GERD & $26(32.5)$ & $51(17.3)$ & $77(20.5)$ \\
\hline
\end{tabular}

the outcomes of interest with officers and non-Caucasian subjects with a decreased risk of IBS, but an increased risk of functional constipation. After adjustment for covariates (Table 3), only IBS retained a significant association with giardiasis (aRR: 2.1, 95 \% CI: 1.1, 4.0).

\section{Discussion}

This study demonstrated over a 2-fold risk of IBS among those with an antecedent Giardia infection and an unexposed reference population after adjusting for covariates.
While several studies have described the incidence of FGD following bacterial and viral IGE few have examined their incidence subsequent to parasitic infection $[8,14]$.

The incidence of Giardia reported here is lower than recorded in another recently published study in this population. Specifically, the Medical Surveillance Monthly Report (MSMR) published in October of 2013 cites an 11-year surveillance period of the US Armed Forces for gastrointestinal infections [15]. The authors reported an incidence rate of giardiasis of 6.2 per 100,000 . The study reported here utilized data from 1998 to 2009, while the MSMR focused on 2002-2012 [15]. The highest rates of Giardia from the MSMR study were in 2002-2006 and from 2008 to 2012 [15]. Case diagnosis year was not available, precluding annual comparisons across the two studies. One potential reason for the difference in incidence estimates could be variable inclusion criteria. For inclusion in this study, a subject could not have a prior documented medical encounter with any of the outcomes of interest (IBS, GERD, constipation, dyspepsia) to help cut down on the likelihood that the outcome of interest could be attributed to something other than the parasitic exposure. If a participant had a prior medical encounter with one of the outcomes of interest it would underestimate the incidence.

The results of this study are consistent with other studies reporting an increase in IBS after enteric infection [16-18]. While not specific to parasitic infections, two recent meta-analyses have shown that the risk increases 6- to 7-fold after IGE and remains elevated for at least 2 to 3 years after the initial infection $[9,10]$. In only one prior study has antecedent Giardia infection been linked to IBS. Following a Giardia outbreak in Bergen, Norway, investigators found a $46.1 \%$ IBS prevalence in exposed subjects $(46.1 \%)$ compared to $14.0 \%$ in the control subjects (aRR: 3.4, 95 \% CI: 2.9, 3.8) [17]. Similarly, we found a 2.1 increase risk in IBS following sporadic Giardia infections compared to an uninfected reference population. The estimate reported here (aRR: 2.1 ) is lower than that reported in prior systematic reviews or in the prior G. lamblia outbreak possibly due to case definitions, study design and/or differences in study populations. The Bergen study used a questionnaire mailed to participants with standardized questions where IBS was defined according to the Rome III criteria, whereas we relied on passive surveillance through DoD medical encounters [17].

Evidence of the association between acute IGE and development of functional dyspepsia has been accumulating in the literature over the past decade. The findings of this study support the growing body of evidence demonstrating an association between acute GI infection and functional dyspepsia [16, 18]. FGD was the third 
Table 2 Unadjusted incident rate ratios (95\% confidence intervals) for outcomes of interest and selected covariates evaluating risk of functional gastrointestinal disorders following acute G. lamblia infection

\begin{tabular}{|c|c|c|c|c|}
\hline \multirow[t]{2}{*}{ Covariate } & \multicolumn{4}{|c|}{ Outcome of interest } \\
\hline & $\mathrm{IBS}$ & GERD & Dyspepsia & Constipation \\
\hline Non-Caucasian & $0.5(0.2,1.4)$ & $1.2(0.8,1.8)$ & $0.9(0.3,3.0)$ & $0.4(0.1,2.9)$ \\
\hline$>$ High school education & $1.5(0.8,3.0)$ & $0.9(0.6,1.3)$ & $0.8(0.2,2.7)$ & $1.7(0.5,5.7)$ \\
\hline Officer & $1.6(0.7,3.3)$ & $1.3(0.9,2.0)$ & $1.8(0.6,5.1)$ & $5.4(0.7,43.5)$ \\
\hline Non-Army Service & $1.8(0.7,4.4)$ & $1.5(1.0,2.4)$ & $2.3(0.6,9.8)$ & $1.7(0.4,7.6)$ \\
\hline Operational deployment & $2.0(0.7,5.6)$ & $1.0(0.5,2.2)$ & $0.4(0.1,3.3)$ & $0.7(0.1,7.6)$ \\
\hline Axis I disorder & $6.7(3.7,12.2)$ & $4.0(2.9,5.6)$ & $5.4(1.7,16.5)$ & $18.4(4.2,81.1)$ \\
\hline Bacterial or Viral Infection & $5.9(3.2,10.9)$ & $2.1(1.3,3.5)$ & $3.8(1.0,15.2)$ & $9.0(2.7,29.8)$ \\
\hline G. lamblia & $2.1(1.1,3.7)$ & $1.1(0.7,1.6)$ & $3.2(1.2,8.9)$ & $1.3(0.4,4.8)$ \\
\hline
\end{tabular}

most prevalent FGD found within the study population (overall estimate of $3.5 \%$ ); however, this is probably an underestimate. Most studies show the prevalence of post-infectious FD around $10 \%$ [11]. Quigley and Lacy reported a prevalence of $12-15 \%$ among the general US population [19]. A meta-analysis in 2013 estimated a pooled OR of 2.18 (95\% CI: 1.70, 2.81) for the risk of developing FD following IGE [12]. Dizdar recently reported that patients with abdominal symptoms after acute Giardia infection had evidence of enhanced visceral sensitivity, and 15 of the 22 patients had evidence of FD indicating that dyspepsia may be attributed to increased visceral sensitivity that persists after initial infection [16]. A recent study [16].

This study found a similar trend with more cases having a GERD diagnosis (32.5 \%) compared to controls $(17.3 \%)$; however, this was not significant in multivariate analyses. A 2012 study looking at three recent norovirus outbreaks found the risk of GERD is higher among those who had an IGE diagnosis during a confirmed outbreak (aRR: $1.39,95$ \% CI: 1.07, 1.81) [20]. The potential association between Giardia and functional dyspepsia needs further study.

The mechanisms by which Giardia infection may induce chronic, long-term FGD are unclear. Recently, researchers at the University of Calgary have shown that infection with Giardia can induce functional changes in commensal flora composition in a nematode model [21]. In a healthy host, it may be that these infections trigger the intestinal dysbiosis and altered sensory perception common noted in many FGDs [22]. Additionally, Giardia has been shown to disrupt tight junctions, which perhaps alters gut permeability in a way that alters normal homeostasis and host-microbiota interactions [23]. The link between giardiasis and post-infectious sequelae is likely the result of a multi-factorial process in a susceptible host and understanding these processes and host characteristics is an ongoing area of research across multiple institutions.

Psychological comorbidities, such as stress and anxiety, are associated with an increased IBS risk, and often the two conditions occur together [14, 24, 25]. In fact, we observed a stronger association between axis I disorders and FGDs then was observed with our exposure of interest (ie, Giardia infection). Several studies have demonstrated a similar association between axis I disorders and IBS $[8,9,24,25]$. Additionally, traumatic life events can often precede the onset of functional bowel disorders [26]. A meta-analysis conducted in 2002 found that psychiatric comorbidities were present in up to $94 \%$ of patients with chronic gastrointestinal disorders, including IBS [27]. We observed an increase in the risk of IBS, GERD, dyspepsia, and constipation in subjects with preceding axis I diagnoses after controlling for relevant covariates and exposures. These results are consistent with observed associations between psychological comorbidities and IBS $[9,10]$. It is important to note, that the associations between giardia and chronic GI disorders

Table 3 Adjusted incident rate ratios (95\% confidence intervals) for outcomes of interest and selected covariates evaluating risk of functional gastrointestinal disorders following acute G. lamblia infection

\begin{tabular}{lllll}
\hline & IBS & GERD & Dyspepsia & Constipation \\
\hline Officer & $0.3(0.1,1.0)$ & $1.7(0.7,3.9)$ & $1.2(0.2,7.8)$ & $6.8(2.0,22.8)$ \\
Non-Caucasian & $0.3(0.1,1.0)$ & $1.9(0.8,4.3)$ & $1.2(0.2,9.7)$ & $2.8(0.6,13.2)$ \\
Axis I disorder & $12.5(4.1,37.7)$ & $3.4(2.4,4.7)$ & $4.6(1.3,16.5)$ & $9.8(2.2,43.7)$ \\
Bacterial or Viral Infection & $2.3(0.8,6.4)$ & $1.3(0.7,2.4)$ & $1.8(0.4,8.2)$ & $2.7(0.7,9.2)$ \\
G. lamblia & $2.1(1.1,4.0)$ & $1.0(0.7,1.4)$ & $2.6(0.9,7.4)$ & $1.2(0.3,5.2)$ \\
\hline
\end{tabular}


were noted to be significant when controlling for these important known covariates. Interactions between IGE and psychological co morbidities were difficult to investigate due to low numbers of each individual comorbidity.

Limitations inherent in our study may impact the generalizability of these results. Giardia infections were identified exclusively among males in this study population, perhaps not unsurprising given the study population and the inclusion criteria. Interactions between covariates were not investigated due to a small sample size of the outcome of interest and relatively low numbers of participants within each subcategory of covariates. FGD diagnoses are complicated by their functional nature and the lack of diagnostic tests [7]. It is also possible that the observed findings could be the result of an unmeasured confounder; however, no confounders have been proposed to date that would differentially affect the exposed cohort. The etiology of enteric infection is also complicated by poor diagnostic test utilization and a low rate of care-seeking behavior in persons with IGE. The utilization of ICD9-CM codes to identify exposures and outcomes can be problematic and has been described elsewhere [28].

\section{Conclusions}

Despite the limitations, this study suggests an increased risk of chronic GI outcomes following Giardiasis similar to what has been reported with other enteric infections. Given recent literature from the FERG on the G. lamblia-specific global morbidity [5] and the fact that Giardia is a major contributor to food and waterborne illnesses in the United States [2], understanding the association between acute infection and these chronic sequelae is important and will yield more accurate estimates of global disease morbidity.

\section{Acknowledgements}

The authors would like to thank the staff of the Armed Forces Health Surveillance Branch for data compilation and provision.

\section{Funding}

This study was funded in part by the Military Infectious Disease Research Program.

\section{Availability of data and materials}

The data that support the findings of this study are available with permission of Armed Forces Health Surveillance Branch.

\section{Authors' contributions}

CP conceived of and participated in the design of the study, assisted in the statistical analyses and helped to draft the manuscript. RG conceived of and participated in the design of the study. MD performed the statistical analyses and helped to draft the manuscript. All authors read and approved the final manuscript.

\section{Competing interests}

The authors declare they have no competing interests.

\section{Ethics approval and consent to participate}

The study protocol was approved by the Naval Medical Research Center Institutional Review Board as an Exempt study in compliance with all applicable Federal regulations governing the protection of human subjects.

\section{Disclaimer}

The views expressed in this article are those of the author and do not necessarily reflect the official policy or position of the Department of the Navy, Department of Defense, nor the U.S. Government. This is a US Government work. There are no restrictions on its use. There were no financial conflicts of interests among any of the authors.

\section{Copyright statement}

Two of the authors are employees of the U.S. Government or military service members. This work was prepared as part of official duties. Title 17 U.S.C. $\S 105$ provides that 'Copyright protection under this title is not available for any work of the United States Government.' Title 17 U.S.C. \$101 defines a U.S. Government work as a work prepared by a military service member or employee of the U.S. Government as part of that person's official duties.

\section{Author details}

${ }^{1}$ Enteric Disease Department, Naval Medical Research Center, 503 Robert Grant Avenue, Silver Spring, MD 20910, USA. ${ }^{2}$ George Washington University, Washington, USA.

Received: 10 March 2016 Accepted: 10 August 2016

Published online: 30 August 2016

\section{References}

1. Farthing MJ. Giardiasis. Gastroenterol Clin North Am. 1996;25(3):493-515.

2. Scallan $E$, et al. Foodborne illness acquired in the United States-major pathogens. Emerg Infect Dis. 2011;17(1):7-15.

3. Kasper MR, et al. Diarrhea outbreak during U.S. military training in E Salvador. PLoS One. 2012;7(7):e40404.

4. Sharp TW, et al. Diarrheal disease among military personnel during Operation Restore Hope, Somalia, 1992-1993. Am J Trop Med Hyg. 1995:52(2):188-93.

5. Torgerson PR, World Health Organization, et al. Estimates of the Global and Regional Disease Burden of 11 Foodborne Parasitic Diseases, 2010: A Data Synthesis. PLoS Med. 2015;12(12):e1001920.

6. Corazziari E. Definition and epidemiology of functional gastrointestinal disorders. Best Pract Res Clin Gastroenterol. 2004;18(4):613-31.

7. Agreus L. The epidemiology of functional gastrointestinal disorders. Eur J Surg Suppl. 1998;583:60-6.

8. Porter CK, et al. The Incidence and Gastrointestinal Infectious Risk of Functional Gastrointestinal Disorders in a Healthy US Adult Population. Am J Gastroenterol. 2011;106(1):130-8.

9. Halvorson HA, Schlett CD, Riddle MS. Postinfectious irritable bowel syndrome-a meta-analysis. Am J Gastroenterol. 2006;101(8):1894-9. quiz 1942.

10. Thabane M, Kottachchi DT, Marshall JK. Systematic review and metaanalysis: The incidence and prognosis of post-infectious irritable bowel syndrome. Aliment Pharmacol Ther. 2007;26(4):535-44.

11. Futagami $S$, Itoh $T$, Sakamoto C. Systematic review with meta-analysis: post-infectious functional dyspepsia. Aliment Pharmacol Ther. 2015;41(2):177-88.

12. Pike $B L$, et al. Acute gastroenteritis and the risk of functional dyspepsia: a systematic review and meta-analysis. Am J Gastroenterol. 2013;108(10): 1558-63. quiz 1564

13. Zou G. A modified poisson regression approach to prospective studies with binary data. Am J Epidemiol. 2004;159(7):702-6.

14. Trivedi $\mathrm{KH}$, et al. The impact of post-infectious functional gastrointestinal disorders and symptoms on the health-related quality of life of US military personnel returning from deployment to the Middle East. Dig Dis Sci. 2011;56(12):3602-9.

15. Armed Forces Health Surveillance, C. Gastrointestinal infections, active component, U.S. Armed Forces, 2002-2012. MSMR. 2013;20(10):7-11. discussion 11

16. Dizdar V, Gilja OH, Hausken T. Increased visceral sensitivity in Giardiainduced postinfectious irritable bowel syndrome and functional dyspepsia. Effect of the 5HT3-antagonist ondansetron. Neurogastroenterol Motil. 2007; 19(12):977-82 
17. Wensaas KA, et al. Irritable bowel syndrome and chronic fatigue 3 years after acute giardiasis: historic cohort study. Gut. 2012;61(2):214-9.

18. Halliez MC, Buret AG. Extra-intestinal and long term consequences of Giardia duodenalis infections. World J Gastroenterol. 2013;19(47):8974-85.

19. Quigley EM, Lacy BE. Overlap of functional dyspepsia and GERD-diagnostic and treatment implications. Nat Rev Gastroenterol Hepatol. 2013;10(3):175-86.

20. Porter CK, et al. Postinfectious gastrointestinal disorders following norovirus outbreaks. Clin Infect Dis. 2012;55(7):915-22.

21. Gerbaba TK, et al. Giardia duodenalis -induced alterations of commensal bacteria kill Caenorhabditis elegans: A new model to study microbial microbial interactions in the gut. Am J Physiol Gastrointest Liver Physiol. 2015;308:G550-61

22. Aguilera M, Cerda-Cuellar M, Martinez V. Antibiotic-induced dysbiosis alters host-bacterial interactions and leads to colonic sensory and motor changes in mice. Gut Microbes. 2015:6(1):10-23.

23. Beatty JK, Bhargava A, Buret AG. Post-infectious irritable bowel syndrome: Mechanistic insights into chronic disturbances following enteric infection. World J Gastroenterol. 2014;20(14):3976-85.

24. Gwee KA, et al. Psychometric scores and persistence of irritable bowel after infectious diarrhoea. Lancet. 1996;347(8995):150-3.

25. Tuteja AK, et al. Development of functional diarrhea, constipation, irritable bowel syndrome, and dyspepsia during and after traveling outside the USA Dig Dis Sci. 2008:53(1):271-6.

26. Naess $\mathrm{H}$, et al. Chronic fatique syndrome after Giardia enteritis: clinical characteristics, disability and long-term sickness absence. BMC Gastroenterol. 2012:12:13

27. Whitehead WE, Palsson O, Jones KR. Systematic review of the comorbidity of irritable bowel syndrome with other disorders: what are the causes and implications? Gastroenterology. 2002;122(4):1140-56.

28. Smith B, et al. Challenges of self-reported medical conditions and electronic medical records among members of a large military cohort. BMC Med Res Methodol. 2008;8:37.

\section{Submit your next manuscript to BioMed Central and we will help you at every step:}

- We accept pre-submission inquiries

- Our selector tool helps you to find the most relevant journal

- We provide round the clock customer support

- Convenient online submission

- Thorough peer review

- Inclusion in PubMed and all major indexing services

- Maximum visibility for your research

Submit your manuscript at www biomedcentral.com/submit

) Biomed Central 